\title{
MAGNETOELECTRIC PONDEROMOTIVE FORCE
}

\author{
SYLVAIN D. BRECHET*, ALEXANDRE ROULET and JEAN-PHILIPPE ANSERMET ${ }^{\dagger}$ \\ Institute of Condensed Matter Physics, \\ Station 3, EPFL, 1015 Lausanne, Switzerland \\ *sylvain.brechet@epfl.ch \\ ${ }^{\dagger}$ jean-philippe.ansermet@epfl.ch
}

Received 6 May 2013

Accepted 7 June 2013

Published 5 August 2013

\begin{abstract}
The dynamics of a system consisting of a matter continuum with a weak linear magnetoelectric coupling interacting with electromagnetic fields is examined on a local scale in a nonrelativistic limit. A consistent expression for the internal energy of the system is derived. The internal energy density and the continuity equation for the momentum lead to the derivation of ponderomotive forces. A nonuniform magnetoelectric coupling generates a "magnetoelectric" ponderomotive force that could be distinguished from the purely electric or magnetic ponderomotive forces by applying alternating electric and magnetic fields at distinct frequencies.
\end{abstract}

Keywords: Magnetoelectricity.

\section{Introduction}

A ponderomotive force results from the response of inhomogeneous matter fields to the presence of electromagnetic fields. Ponderomotive forces are generally overlooked since the electromagnetic community is not much concerned with continuum mechanics and the continuum mechanics community is not dealing usually with electromagnetic systems.

In this paper, we determine the analytical expressions of the ponderomotive forces for a system consisting of a matter continuum with a weak linear magnetoelectric coupling interacting with electromagnetic fields. The electrodynamics of continuous media with a magnetoelectric coupling is discussed notably by O'Dell ${ }^{1}$ and Serdyukov et al. ${ }^{2}$ Magnetoelectric effects are currently of interest because they appear in topological insulators, which exhibit, as was shown by Zhang et al., ${ }^{3}$ a linear magnetoelectric coupling in the electromagnetic constitutive relations. The magnetoelectric coupling found in multiferroics are reviewed notably by Khomskii ${ }^{4}$ and Fiebig. ${ }^{5}$ In the presence of a linear magnetoelectric coupling, great care has to 
be taken in the choice of electromagnetic state fields. In particular, the linear constitutive equations have to be defined carefully in order to be invertible and to lead to a consistent dynamical analysis yielding a correct expression for the ponderomotive forces.

In order to obtain an explicit expression for the ponderomotive forces, we split the dynamics of the system, given by the continuity equation for the momentum, into a matter part and an electromagnetic part that contains the contribution of the electromagnetic fields and of their interaction with the matter fields. The dynamics is described in the local rest frame of the matter continuum referred to as the local material frame. In most experimental conditions, the matter drift velocity is clearly nonrelativistic. Thus, we restrict our analysis to the nonrelativistic limit. This nonrelativistic treatment shows that Minkowski's definition ${ }^{6}$ of the electromagnetic momentum leads to a consistent expression for the ponderomotive forces.

\section{Electromagnetic Linear Constitutive Relations}

The state of a system consisting of a matter continuum interacting with linear electromagnetic fields is described locally by a set of state fields. ${ }^{7,8}$ These fields are the matter density field $n$, the matter velocity field $\mathbf{v}$, the strain tensor $\mathbf{u}$, the electric displacement field $\mathbf{D}$ and the magnetic induction field $\mathbf{B}$. Note that the velocity field $\mathbf{v}$ vanishes for solids at rest and the strain tensor $\mathbf{u}$ vanishes for fluids. The electromagnetic fields $\mathbf{D}$ and $\mathbf{B}$ are frame-independent in the nonrelativistic limit, i.e. $\mathbf{v}^{2} / c^{2} \rightarrow 0$, where $c$ represents the speed of light in the vacuum. ${ }^{9}$

The electromagnetic constitutive relations relate the electromagnetic fields representing densities of extensive electromagnetic properties to the electromagnetic fields corresponding to intensive electromagnetic properties. The extensive electromagnetic properties are the electric dipoles and the magnetic moments of the physical system. The corresponding densities are the electric polarization and the magnetization.

In the presence of a magnetoelectric coupling in a linear electromagnetic framework, the total electric polarization is given by the electric displacement field $\mathbf{D}(n, \mathbf{u}, \mathbf{E}, \mathbf{B})$, that is a function of the matter density $n$ and the strain tensor $\mathrm{u}$, and a linear mapping of the electric field $\mathbf{E}$ and the magnetic induction field $\mathbf{B}$. Note that the electromagnetic fields $\mathbf{E}$ and $\mathbf{B}$ are intensive fields. The total electric polarization is split into the electric polarization of the vacuum and the electric polarization of the matter, i.e.

$$
\mathbf{D}(n, \mathrm{u}, \mathbf{E}, \mathbf{B})=\varepsilon_{0} \mathbf{E}+\mathbf{P}(n, \mathrm{u}, \mathbf{E}, \mathbf{B}),
$$

where $\varepsilon_{0}$ is the vacuum permittivity and $\mathbf{P}(n, \mathbf{u}, \mathbf{E}, \mathbf{B})$ is the electric polarization of the matter. In the absence of a spontaneous electric polarization, the electric polarization of the matter $\mathbf{P}(n, \mathbf{u}, \mathbf{E}, \mathbf{B})$ is linearly induced by the electric field $\mathbf{E}$ 
and the magnetic induction field $\mathbf{B}$, i.e.

$$
\mathbf{P}(n, \mathrm{u}, \mathbf{E}, \mathbf{B})=\varepsilon_{0} \chi_{e}(n, \mathrm{u}) \cdot \mathbf{E}+c \varepsilon_{0} \chi_{\alpha}(n, \mathrm{u}) \cdot \mathbf{B},
$$

where $c$ is the speed of light in the vacuum, $\chi_{e}(n, \mathrm{u})$ is the electric susceptibility tensor and $\chi_{\alpha}(n, \mathrm{u})$ is the magnetoelectric susceptibility tensor. Note that these tensors are dimensionless.

In the presence of a magnetoelectric coupling in a linear electromagnetic framework, the total magnetization is given by the opposite of the magnetic field $\mathbf{H}(n, \mathbf{u}, \mathbf{E}, \mathbf{B})$, that is a function of the matter density $n$ and the strain tensor $\mathbf{u}$, and a linear mapping of the electric field $\mathbf{E}$ and the magnetic induction field B. It is split into the magnetization of the vacuum and the magnetization of the matter, i.e.

$$
-\mathbf{H}(n, \mathrm{u}, \mathbf{E}, \mathbf{B})=-\mu_{0}^{-1} \mathbf{B}+\mathbf{M}(n, \mathbf{u}, \mathbf{E}, \mathbf{B}),
$$

where $\mathbf{M}(n, \mathbf{u}, \mathbf{E}, \mathbf{B})$ is the magnetization of the matter and $\mu_{0}$ is the vacuum, which satisfies $c^{-2}=\varepsilon_{0} \mu_{0}$. Note that the negative signs in the relation (3) are a consequence of the historical choice of definition for $\mathbf{M}$. In the absence of a spontaneous magnetization, the magnetization of the matter $\mathbf{M}(n, \mathbf{u}, \mathbf{E}, \mathbf{B})$ is linearly induced by the electric field $\mathbf{E}$ and the magnetic induction field $\mathbf{B}$, i.e.

$$
\mathbf{M}(n, \mathrm{u}, \mathbf{E}, \mathbf{B})=c^{-1} \mu_{0}^{-1} \chi_{\alpha}(n, \mathrm{u}) \cdot \mathbf{E}+\mu_{0}^{-1} \chi_{m}(n, \mathrm{u}) \cdot \mathbf{B},
$$

where $\chi_{m}(n, \mathrm{u})$ is the magnetic susceptibility tensor. Note that the same magnetoelectric susceptibility tensor $\chi_{\alpha}(n, \mathrm{u})$ appears in the expressions (2) and (4) for the electric polarization and the magnetization of the matter, respectively. This is due to the fact that $\chi_{\alpha}(n, \mathrm{u})$ represents geometric properties of the matter.

The electric permittivity tensor $\varepsilon(n, \mathbf{u})$, the magnetic permeability tensor $\boldsymbol{\mu}(n, \mathbf{u})$ and the magnetoelectric coupling tensor $\boldsymbol{\alpha}(n, \mathrm{u})$ are respectively defined as

$$
\begin{aligned}
\varepsilon(n, \mathrm{u}) & =\varepsilon_{0}\left(\mathbb{1}+\chi_{e}(n, \mathrm{u})\right), \\
\boldsymbol{\mu}(n, \mathrm{u}) & =\mu_{0}\left(\mathbb{1}-\chi_{m}(n, \mathrm{u})\right)^{-1}=c^{-2} \varepsilon_{0}^{-1}\left(\mathbb{1}-\chi_{m}(n, \mathrm{u})\right)^{-1}, \\
\boldsymbol{\alpha}\left(s, n_{A}\right) & =c \varepsilon_{0} \boldsymbol{\chi}_{\alpha}(n, \mathrm{u})=c^{-1} \mu_{0}^{-1} \chi_{\alpha}(n, \mathrm{u}),
\end{aligned}
$$

where $\mathbb{1}$ is the identity rank-2 tensor. Using definitions (5), the electric constitutive relation (1) and the magnetic constitutive relation (3) are recast respectively as

$$
\begin{aligned}
& \mathbf{D}(n, \mathrm{u}, \mathbf{E}, \mathbf{B})=\varepsilon(n, \mathrm{u}) \cdot \mathbf{E}+\boldsymbol{\alpha}(n, \mathrm{u}) \cdot \mathbf{B}, \\
& \mathbf{H}(n, \mathrm{u}, \mathbf{E}, \mathbf{B})=\boldsymbol{\alpha}(n, \mathrm{u}) \cdot \mathbf{E}+\boldsymbol{\mu}^{-1}(n, \mathrm{u}) \cdot \mathbf{B} .
\end{aligned}
$$

Since the electric fields $\mathbf{D}$ and $\mathbf{E}$ are vectors and the magnetic fields $\mathbf{H}$ and $\mathbf{B}$ are pseudo-vectors, the invariance of the constitutive relations (9) under inversion implies that the phenomenological tensors $\boldsymbol{\varepsilon}(n, \mathrm{u})$ and $\boldsymbol{\mu}(n, \mathrm{u})$ are symmetric and 
the phenomenological tensor $\boldsymbol{\alpha}(n, \mathrm{u})$ is antisymmetric, i.e.

$$
\begin{aligned}
\varepsilon^{T}(n, \mathrm{u}) & =\boldsymbol{\varepsilon}(n, \mathrm{u}), \\
\boldsymbol{\mu}^{T}(n, \mathrm{u}) & =\boldsymbol{\mu}(n, \mathrm{u}), \\
\boldsymbol{\alpha}^{T}(n, \mathrm{u}) & =-\boldsymbol{\alpha}(n, \mathrm{u}),
\end{aligned}
$$

where the exponent $T$ denotes the transpose. Since the tensor $\boldsymbol{\alpha}(n, \mathrm{u})$ is antisymmetric, the electric polarization (2) induced by the magnetic induction field $\mathbf{B}$ and the magnetization (4) induced by the electric field $\mathbf{E}$ can be recast in terms of the dual vector $\hat{\boldsymbol{\alpha}}(n, \mathrm{u})$ of $\boldsymbol{\alpha}(n, \mathrm{u})$, i.e.

$$
\begin{aligned}
\mathbf{P}(n, \mathrm{u}, \mathbf{0}, \mathbf{B}) & =\boldsymbol{\alpha}(n, \mathrm{u}) \cdot \mathbf{B}=\hat{\boldsymbol{\alpha}}(n, \mathrm{u}) \times \mathbf{B}, \\
\mathbf{M}(n, \mathrm{u}, \mathbf{E}, \mathbf{0}) & =\boldsymbol{\alpha}(n, \mathrm{u}) \cdot \mathbf{E}=\hat{\boldsymbol{\alpha}}(n, \mathrm{u}) \times \mathbf{E} .
\end{aligned}
$$

This implies that $\mathbf{P}(n, \mathbf{u}, \mathbf{0}, \mathbf{B})$ is orthogonal to $\mathbf{B}$ and $\mathbf{M}(n, \mathbf{u}, \mathbf{E}, \mathbf{0})$ is orthogonal to $\mathbf{E}$.

Experimentally, magnetoelectric effects are known to be much smaller than electromagnetic effects. The weakness of the magnetoelectric coupling is expressed in terms of the phenomenological tensors as

$$
\boldsymbol{\alpha}^{2}(n, \mathrm{u}) \ll \varepsilon^{-1}(n, \mathrm{u}) \cdot \boldsymbol{\mu}(n, \mathrm{u}) .
$$

Under the weak coupling condition (8), the inverse of the electromagnetic constitutive relations (6) are expressed in terms of the fields $n, \mathbf{u}, \mathbf{D}$ and $\mathbf{H}$ as

$$
\begin{aligned}
& \mathbf{E}(n, \mathbf{u}, \mathbf{D}, \mathbf{H})=\varepsilon^{-1}(n, \mathrm{u}) \cdot \mathbf{D}-\left(\varepsilon^{-1}(n, \mathrm{u}) \cdot \boldsymbol{\alpha}(n, \mathrm{u}) \cdot \boldsymbol{\mu}(n, \mathrm{u})\right) \cdot \mathbf{H}, \\
& \mathbf{B}(n, \mathrm{u}, \mathbf{D}, \mathbf{H})=-\left(\boldsymbol{\mu}(n, \mathrm{u}) \cdot \boldsymbol{\alpha}(n, \mathrm{u}) \cdot \boldsymbol{\varepsilon}^{-1}(n, \mathrm{u})\right) \cdot \mathbf{D}+\boldsymbol{\mu}(n, \mathrm{u}) \cdot \mathbf{H} .
\end{aligned}
$$

Under the same condition, the electromagnetic constitutive relations (6) and (9) are recast in terms of the fields $n, \mathbf{u}, \mathbf{D}$ and $\mathbf{B}$ as

$$
\begin{aligned}
\mathbf{E}(n, \mathrm{u}, \mathbf{D}, \mathbf{B}) & =\varepsilon^{-1}(n, \mathrm{u}) \cdot \mathbf{D}-\left(\varepsilon^{-1}(n, \mathbf{u}) \cdot \boldsymbol{\alpha}(n, \mathrm{u})\right) \cdot \mathbf{B}, \\
\mathbf{H}(n, \mathrm{u}, \mathbf{D}, \mathbf{B}) & =\left(\boldsymbol{\alpha}(n, \mathrm{u}) \cdot \varepsilon^{-1}(n, \mathrm{u})\right) \cdot \mathbf{D}+\boldsymbol{\mu}^{-1}(n, \mathrm{u}) \cdot \mathbf{B},
\end{aligned}
$$

and in terms of the fields $n, \mathbf{u}, \mathbf{E}$ and $\mathbf{H}$ as

$$
\begin{aligned}
& \mathbf{D}(n, \mathrm{u}, \mathbf{E}, \mathbf{H})=\boldsymbol{\varepsilon}(n, \mathrm{u}) \cdot \mathbf{E}+(\boldsymbol{\alpha}(n, \mathbf{u}) \cdot \boldsymbol{\mu}(n, \mathbf{u})) \cdot \mathbf{H}, \\
& \mathbf{B}(n, \mathbf{u}, \mathbf{E}, \mathbf{H})=-(\boldsymbol{\mu}(n, \mathbf{u}) \cdot \boldsymbol{\alpha}(n, \mathbf{u})) \cdot \mathbf{E}+\boldsymbol{\mu}(n, \mathbf{u}) \cdot \mathbf{H}
\end{aligned}
$$

\section{Local Dynamics}

The local dynamics of the electromagnetic fields is described by Maxwell's equations. These equations consist of two spatial constraint equations and two continuity equations. The constraint equations are Gauss' law and Thomson's law, respectively given by

$$
\begin{aligned}
& \nabla \cdot \mathbf{D}=0, \\
& \nabla \cdot \mathbf{B}=0,
\end{aligned}
$$


for an electrically insulating matter continuum. The continuity equations are Ampère-Maxwell's law and Faraday's law, which are expressed in an Eulerian frame where the matter has a velocity $\mathbf{v}$ as

$$
\begin{aligned}
& \partial_{t} \mathbf{D}=\nabla \times \mathbf{H}^{\prime}, \\
& \partial_{t} \mathbf{B}=-\nabla \times \mathbf{E}^{\prime},
\end{aligned}
$$

where $\mathbf{E}^{\prime}$ and $\mathbf{H}^{\prime}$ are respectively the electric and magnetic fields in that frame. Note that the electric displacement field $\mathbf{D}$ and the magnetic induction field $\mathbf{B}$ are the dynamic fields in Maxwell's equation (14) and Faraday's equation (15), respectively whereas the electric field $\mathbf{E}^{\prime}$ and the magnetic field $\mathbf{H}^{\prime}$ are merely auxiliary fields. Therefore, we choose $\mathbf{D}$ and $\mathbf{B}$ as state fields.

In the nonrelativistic limit, the transformation laws relating the electric field $\mathbf{E}^{\prime}$ and magnetic field $\mathbf{H}^{\prime}$ in the Eulerian frame where the matter has a velocity $\mathbf{v}$ to the electric field $\mathbf{E}$ and magnetic field $\mathbf{H}$ in the local Lagrangian frame where the matter is at rest, i.e. $\mathbf{v}=\mathbf{0}$, are given by ${ }^{9}$

$$
\begin{aligned}
\mathbf{E}^{\prime}(n, \mathbf{u}, \mathbf{v}, \mathbf{D}, \mathbf{B}) & =\mathbf{E}(n, \mathbf{u}, \mathbf{D}, \mathbf{B})-\mathbf{v} \times \mathbf{B}, \\
\mathbf{H}^{\prime}(n, \mathbf{u}, \mathbf{v}, \mathbf{D}, \mathbf{B}) & =\mathbf{H}(n, \mathbf{u}, \mathbf{D}, \mathbf{B})+\mathbf{v} \times \mathbf{D} .
\end{aligned}
$$

Using Gauss' law (12), Thomson's law (13), the transformation laws (16) and the definition of the time differential operator in the local material frame, i.e.

$$
\cdot \equiv \partial_{t}+\mathbf{v} \cdot \nabla
$$

Ampère-Maxwell's law (14) and Faraday's law (15) are respectively recast in the local material frame as

$$
\begin{aligned}
& \dot{\mathbf{D}}+(\boldsymbol{\nabla} \cdot \mathbf{v}) \mathbf{D}-(\mathbf{D} \cdot \boldsymbol{\nabla}) \mathbf{v}=\boldsymbol{\nabla} \times \mathbf{H}, \\
& \dot{\mathbf{B}}+(\boldsymbol{\nabla} \cdot \mathbf{v}) \mathbf{B}-(\mathbf{B} \cdot \boldsymbol{\nabla}) \mathbf{v}=-\boldsymbol{\nabla} \times \mathbf{E},
\end{aligned}
$$

and hold only in the nonrelativistic limit.

The internal energy density $u(n, \mathbf{u}, \mathbf{D}, \mathbf{B})$ defined as the energy density in the local material frame, where the local element of continuum is a rest, i.e. $\mathbf{v}=\mathbf{0}$, can be split as

$$
u(n, \mathrm{u}, \mathbf{D}, \mathbf{B})=u^{\mathrm{mat}}(n, \mathrm{u})+u^{\mathrm{em}}(n, \mathrm{u}, \mathbf{D}, \mathbf{B}),
$$

where $u^{\text {mat }}(n, \mathbf{u}) \equiv u(n, \mathbf{u}, \mathbf{0}, \mathbf{0})$ is the internal energy density of the matter fields and $u^{\mathrm{em}}(n, \mathbf{u}, \mathbf{D}, \mathbf{B})$ is the internal energy density of the electromagnetic fields and of their interaction with the matter fields.

The electromagnetic state fields $\mathbf{E}$ and $\mathbf{H}$ are defined as the conjugate fields of the electromagnetic state fields $\mathbf{D}$ and $\mathbf{B}$, respectively, i.e.

$$
\begin{aligned}
\mathbf{E}(n, \mathbf{u}, \mathbf{D}, \mathbf{B}) & \equiv \frac{\partial u(n, \mathbf{u}, \mathbf{D}, \mathbf{B})}{\partial \mathbf{D}}, \\
\mathbf{H}(n, \mathbf{u}, \mathbf{D}, \mathbf{B}) & \equiv \frac{\partial u(n, \mathbf{u}, \mathbf{D}, \mathbf{B})}{\partial \mathbf{B}} .
\end{aligned}
$$


The analytical expression for the electromagnetic part of the internal energy density $u^{\mathrm{em}}(n, \mathrm{u}, \mathbf{D}, \mathbf{B})$ has to satisfy the integrability conditions (21) and the symmetries of the phenomenological tensors that appear in the constitutive equations (10), i.e.

$$
\begin{aligned}
u^{\mathrm{em}}(n, \mathrm{u}, \mathbf{D}, \mathbf{B})= & \frac{1}{2}\left(\varepsilon^{-1}\left(s, n_{A}\right) \cdot(\mathbf{D} \odot \mathbf{D})+\boldsymbol{\mu}^{-1}\left(s, n_{A}\right) \cdot(\mathbf{B} \odot \mathbf{B})\right) \\
& -\left(\varepsilon^{-1}\left(s, n_{A}\right) \cdot \boldsymbol{\alpha}\left(s, n_{A}\right)\right) \cdot(\mathbf{D} \otimes \mathbf{B})
\end{aligned}
$$

where the symbols $\odot$ and $\otimes$ denote respectively a symmetrized and an antisymmetrized tensorial product. Using the constitutive relations (10), the electromagnetic part of the internal energy density (22) is recast as

$$
u^{\mathrm{em}}(n, \mathrm{u}, \mathbf{D}, \mathbf{B})=\frac{1}{2}(\mathbf{E}(n, \mathrm{u}, \mathbf{D}, \mathbf{B}) \cdot \mathbf{D}+\mathbf{H}(n, \mathbf{u}, \mathbf{D}, \mathbf{B}) \cdot \mathbf{B}) .
$$

The electromagnetic part of the internal energy density (22) can be expressed as a quadratic form in a six dimensions, i.e.

$$
u^{\mathrm{em}}(n, \mathrm{u}, \mathbf{D}, \mathbf{B})=\frac{1}{2} \varepsilon_{0}^{-1} \chi(n, \mathrm{u}) \cdot\left(\left(\mathbf{D}, \varepsilon_{0} c \mathbf{B}\right) \odot\left(\mathbf{D}, \varepsilon_{0} c \mathbf{B}\right)\right),
$$

where $\left(\mathbf{D}, \varepsilon_{0} c \mathbf{B}\right)$ is a vector in six dimensions and $\chi(n, \mathrm{u})$ is a rank-2 dimensionless tensor in six dimensions that is expressed in blocks as

$$
\chi(n, \mathrm{u})=\left(\begin{array}{cc}
\varepsilon_{0} \varepsilon^{-1}(n, \mathrm{u}) & -c^{-1} \varepsilon^{-1}(n, \mathrm{u}) \cdot \boldsymbol{\alpha}(n, \mathrm{u}) \\
c^{-1} \boldsymbol{\alpha}(n, \mathrm{u}) \cdot \varepsilon^{-1}(n, \mathrm{u}) & \mu_{0} \boldsymbol{\mu}^{-1}(n, \mathrm{u})
\end{array}\right) .
$$

Since the electromagnetic part of the internal energy density $u^{\mathrm{em}}(n, \mathbf{u}, \mathbf{D}, \mathbf{B})$ can be expressed as a positive-definite quadratic form (24), it guarantees the existence of a minimum of $u^{\mathrm{em}}(n, \mathrm{u}, \mathbf{D}, \mathbf{B})$ under a variation of the dynamical electromagnetic fields $\mathbf{D}$ and $\mathbf{B}$. Thus, the expression (22) of the electromagnetic part of the internal energy density is the unique physically consistent solution in the presence of a magnetoelectric coupling in a linear electromagnetic framework.

The local time evolution of the momentum is given by the continuity equation,

$$
\dot{\mathbf{p}}+(\boldsymbol{\nabla} \cdot \mathbf{v}) \mathbf{p}-\nabla \cdot \boldsymbol{\sigma}=\mathbf{f}^{\mathrm{ext}},
$$

where $\mathbf{p}(n, \mathbf{v}, \mathbf{D}, \mathbf{B})$ is the momentum density and $\boldsymbol{\sigma}(n, \mathbf{u}, \mathbf{v}, \mathbf{D}, \mathbf{B})$ is the stress tensor and $\mathbf{f}^{\text {ext }}$ is the external force density.

The momentum density consists of two parts: ${ }^{10}$ a matter part $\mathbf{p}^{\text {mat }}(n, \mathbf{v})$ and an electromagnetic part $\mathbf{p}^{\mathrm{em}}(\mathbf{D}, \mathbf{B})$, i.e.

$$
\mathbf{p}(n, \mathbf{v}, \mathbf{D}, \mathbf{B})=\mathbf{p}^{\mathrm{mat}}(n, \mathbf{v})+\mathbf{p}^{\mathrm{em}}(\mathbf{D}, \mathbf{B}),
$$

where

$$
\begin{aligned}
& \mathbf{p}^{\text {mat }}(n, \mathbf{v})=m(n) \mathbf{v}, \\
& \mathbf{p}^{\mathrm{em}}(\mathbf{D}, \mathbf{B})=\mathbf{D} \times \mathbf{B} .
\end{aligned}
$$


Note that the definition (28) of the momentum density, which is Minkowski's definition, ${ }^{6}$ accounts for the contributions of the electromagnetic fields in the vacuum and the contributions of the interaction between the electromagnetic fields and the matter fields. In the vacuum, the auxiliary electric permittivity and the magnetic permeability tensors $\varepsilon$ and $\boldsymbol{\mu}$ reduce respectively to $\varepsilon_{0} \mathbb{1}$ and $\mu_{0} \mathbb{1}$, and the auxiliary magnetoelectric tensor $\boldsymbol{\alpha}$ vanishes. Thus, using the constitutive equations (11) and the definition $c^{-2}=\varepsilon_{0} \mu_{0}$, the momentum density (28) in the vacuum reduces to

$$
\mathbf{p}^{\mathrm{em}}(\mathbf{E}, \mathbf{H})=\frac{1}{c^{2}} \mathbf{E} \times \mathbf{H},
$$

which is Abraham's definition of the momentum. ${ }^{11}$

Similarly to the momentum density, the stress tensor $\boldsymbol{\sigma}(n, \mathbf{u}, \mathbf{v}, \mathbf{D}, \mathbf{B})$ consists also of two parts: a matter part $\boldsymbol{\sigma}^{\text {mat }}(n, \mathbf{u}, \mathbf{v})$ and an electromagnetic part $\boldsymbol{\sigma}^{\mathrm{em}}(n, \mathrm{u}, \mathbf{D}, \mathbf{B})$, i.e.

$$
\boldsymbol{\sigma}(n, \mathrm{u}, \mathbf{v}, \mathbf{D}, \mathbf{B})=\boldsymbol{\sigma}^{\mathrm{mat}}(n, \mathrm{u}, \mathbf{v})+\boldsymbol{\sigma}^{\mathrm{em}}(n, \mathrm{u}, \mathbf{D}, \mathbf{B}) .
$$

The continuity equation (25) for the momentum can be split according to

$$
\begin{gathered}
\dot{\mathbf{p}}^{\text {mat }}+(\boldsymbol{\nabla} \cdot \mathbf{v}) \mathbf{p}^{\text {mat }}-\boldsymbol{\nabla} \cdot \boldsymbol{\sigma}^{\text {mat }}=\mathbf{f}^{\text {ext }}+\mathbf{f}^{\mathrm{em}}, \\
\dot{\mathbf{p}}^{\mathrm{em}}+(\boldsymbol{\nabla} \cdot \mathbf{v}) \mathbf{p}^{\mathrm{em}}-\boldsymbol{\nabla} \cdot \boldsymbol{\sigma}^{\mathrm{em}}=-\mathbf{f}^{\mathrm{em}},
\end{gathered}
$$

where $\mathbf{f}^{\mathrm{em}}$ is the electromagnetic force density exerted by the electromagnetic fields on the matter fields. Since the matter fields and the electromagnetic fields are both part of the local system, the electromagnetic force density $\mathbf{f}^{\mathrm{em}}$ is considered as an "internal" force of the local system. Thus, the force density $\mathrm{f}^{\text {ext }}$ represents the external force density of the non-electromagnetic fields.

The scalar product $\left(\dot{\mathbf{p}}^{\mathrm{em}}+(\boldsymbol{\nabla} \cdot \mathbf{v}) \mathbf{p}^{\mathrm{em}}\right) \cdot \mathbf{v}$ is a relativistic correction. Thus, in a nonrelativistic framework, the scalar product of the continuity equation (32) and the velocity vector $\mathbf{v}$ reduces to

$$
\left(\nabla \cdot \sigma^{\mathrm{em}}\right) \cdot \mathbf{v}=\mathbf{f}^{\mathrm{em}} \cdot \mathbf{v} .
$$

The definition (28) of the electromagnetic momentum $\mathbf{p}^{\mathrm{em}}$, Ampère-Maxwell's law (18) and Faraday's law (19) imply that the scalar product $\dot{\mathbf{p}}^{\mathrm{em}} \cdot \mathbf{v}$ satisfies the dynamical equation,

$$
\begin{aligned}
\left((\mathbf{D} \times \mathbf{B})^{\cdot}+2(\boldsymbol{\nabla} \cdot \mathbf{v})(\mathbf{D} \times \mathbf{B})-((\mathbf{D} \cdot \boldsymbol{\nabla}) \mathbf{v}) \times \mathbf{B}-\mathbf{D} \times((\mathbf{B} \cdot \boldsymbol{\nabla}) \mathbf{v})\right) \cdot \mathbf{v} \\
\quad=(-\mathbf{D} \times(\boldsymbol{\nabla} \times \mathbf{E})-\mathbf{B} \times(\boldsymbol{\nabla} \times \mathbf{H})) \cdot \mathbf{v},
\end{aligned}
$$

where the terms on the LHS are relativistic corrections that are neglected in a nonrelativistic framework and the dot on the righthand corner of the first brackets on the first line denotes a material time derivative. Thus, in the nonrelativistic limit, the dynamical equation (34) reduces to

$$
(\mathbf{D} \times(\boldsymbol{\nabla} \times \mathbf{E})+\mathbf{B} \times(\boldsymbol{\nabla} \times \mathbf{H})) \cdot \mathbf{v}=0 .
$$


Using the vectorial identities,

$$
\mathbf{D} \times(\boldsymbol{\nabla} \times \mathbf{E})=\mathbf{D} \nabla \mathbf{E}-(\mathbf{D} \cdot \boldsymbol{\nabla}) \mathbf{E}=\mathbf{D} \nabla \mathbf{E}+(\boldsymbol{\nabla} \cdot \mathbf{D}) \mathbf{E}-\nabla_{\mathbf{D}} \cdot(\mathbf{D} \odot \mathbf{E})
$$

and

$$
\mathbf{B} \times(\boldsymbol{\nabla} \times \mathbf{H})=\mathbf{B} \nabla \mathbf{H}-(\mathbf{B} \cdot \boldsymbol{\nabla}) \mathbf{H}=\mathbf{B} \nabla \mathbf{H}+(\boldsymbol{\nabla} \cdot \mathbf{B}) \mathbf{H}-\nabla_{\mathbf{B}} \cdot(\mathbf{B} \odot \mathbf{H}),
$$

and Gauss' law (12) and Thomson's law (13), Eq. (35) is recast as

$$
\left(\nabla_{\mathbf{D}, \mathbf{B}} \cdot(\mathbf{D} \odot \mathbf{E}+\mathbf{B} \odot \mathbf{H})\right) \cdot \mathbf{v}=(\mathbf{D} \nabla \mathbf{E}+\mathbf{B} \nabla \mathbf{H}) \cdot \mathbf{v},
$$

where the index $\mathbf{D , B}$ denotes that there is a dot product between the covariant differential operator $\boldsymbol{\nabla}$ and the contravariant electromagnetic vector fields $\mathbf{D}$ and B, respectively.

In order to recast the terms $\mathbf{D} \nabla \mathbf{E}$ and $\mathbf{B} \boldsymbol{\nabla H}$ on the RHS of Eq. (36) and obtain explicitly Eq. (33), we need to introduce the Legendre transform of the electromagnetic part of the internal energy density $\ell_{u}^{\mathrm{em}}(n, \mathbf{u}, \mathbf{E}, \mathbf{H})$. The Legendre transform $\ell_{u}^{\mathrm{em}}(n, \mathbf{u}, \mathbf{E}, \mathbf{H})$ is defined as

$$
\ell_{u}^{\mathrm{em}}(n, \mathbf{u}, \mathbf{E}, \mathbf{H}) \equiv \frac{\partial u^{\mathrm{em}}}{\partial \mathbf{D}} \cdot \mathbf{D}+\frac{\partial u^{\mathrm{em}}}{\partial \mathbf{B}} \cdot \mathbf{B}-u^{\mathrm{em}}(n, \mathbf{u}, \mathbf{D}, \mathbf{B}) .
$$

Using the expression (23) for the electromagnetic part of the internal energy density and the differential relations (21), the Legendre transform (37) is found to be

$$
\ell_{u}^{\mathrm{em}}(n, \mathrm{u}, \mathbf{E}, \mathbf{H})=\frac{1}{2}(\mathbf{E} \cdot \mathbf{D}(n, \mathrm{u}, \mathbf{E}, \mathbf{H})+\mathbf{H} \cdot \mathbf{B}(n, \mathbf{u}, \mathbf{E}, \mathbf{H})),
$$

where the integrability conditions are:

$$
\begin{aligned}
\mathbf{D}(n, \mathrm{u}, \mathbf{E}, \mathbf{H}) & =\frac{\partial \ell_{u}^{\mathrm{em}}(n, \mathrm{u}, \mathbf{E}, \mathbf{H})}{\partial \mathbf{E}}, \\
\mathbf{B}\left(s, n_{A}, \mathbf{E}, \mathbf{H}\right) & =\frac{\partial \ell_{u}^{\mathrm{em}}(n, \mathrm{u}, \mathbf{E}, \mathbf{H})}{\partial \mathbf{H}} .
\end{aligned}
$$

Using the electromagnetic constitutive relations (11), the Legendre transform (38) can be recast as

$$
\begin{aligned}
\ell_{u}^{\mathrm{em}}(n, \mathrm{u}, \mathbf{E}, \mathbf{H})= & \frac{1}{2}(\varepsilon(n, \mathrm{u}) \cdot(\mathbf{E} \odot \mathbf{E})+\boldsymbol{\mu}(n, \mathrm{u}) \cdot(\mathbf{H} \odot \mathbf{H})) \\
& +(\boldsymbol{\alpha}(n, \mathrm{u}) \cdot \boldsymbol{\mu}(n, \mathrm{u})) \cdot(\mathbf{E} \otimes \mathbf{H}) .
\end{aligned}
$$

The constitutive relations (11) imply that the gradient of the Legendre transform (40) is of the form,

$$
\begin{aligned}
\nabla \ell_{u}^{\mathrm{em}}= & \mathbf{D} \nabla \mathbf{E}+\mathbf{B} \nabla \mathbf{H}+\frac{1}{2}((\mathbf{E} \odot \mathbf{E}) \boldsymbol{\nabla} \varepsilon+(\mathbf{H} \odot \mathbf{H}) \boldsymbol{\nabla} \boldsymbol{\mu}) \\
& +(\mathbf{E} \otimes \mathbf{H}) \boldsymbol{\nabla}(\boldsymbol{\alpha} \cdot \boldsymbol{\mu}) .
\end{aligned}
$$


Substituting the relation (41) into Eq. (36), the latter reduces to

$$
\begin{aligned}
& \left(\boldsymbol{\nabla}_{\mathbf{D}, \mathbf{B}} \cdot\left(\mathbf{D} \odot \mathbf{E}+\mathbf{B} \odot \mathbf{H}-\ell_{u}^{\mathrm{em}} \mathbb{1}\right)\right) \cdot \mathbf{v} \\
& \quad=\left(-\frac{1}{2}((\mathbf{E} \odot \mathbf{E}) \boldsymbol{\nabla} \boldsymbol{\varepsilon}+(\mathbf{H} \odot \mathbf{H}) \boldsymbol{\nabla} \boldsymbol{\mu})-(\mathbf{E} \otimes \mathbf{H}) \boldsymbol{\nabla}(\boldsymbol{\alpha} \cdot \boldsymbol{\mu})\right) \cdot \mathbf{v} .
\end{aligned}
$$

By identification of Eqs. (36) and (42), the expressions for the electromagnetic stress tenor $\sigma^{\mathrm{em}}$ and the electromagnetic force density $\mathbf{f}^{\mathrm{em}}$ are found to be

$$
\begin{aligned}
\boldsymbol{\sigma}^{\mathrm{em}} & =\mathbf{D} \odot \mathbf{E}+\mathbf{B} \odot \mathbf{H}-\frac{1}{2}(\mathbf{E} \cdot \mathbf{D}+\mathbf{H} \cdot \mathbf{B}) \mathbb{1}, \\
\mathbf{f}^{\mathrm{em}} & =-\frac{1}{2}((\mathbf{E} \odot \mathbf{E}) \boldsymbol{\nabla} \boldsymbol{\varepsilon}+(\mathbf{H} \odot \mathbf{H}) \boldsymbol{\nabla} \boldsymbol{\mu})-(\mathbf{E} \otimes \mathbf{H}) \boldsymbol{\nabla}(\boldsymbol{\alpha} \cdot \boldsymbol{\mu}) .
\end{aligned}
$$

Using the electromagnetic constitutive relations (6), the definitions (5) of the phenomenological tensors, the vectorial relation,

$$
\nabla \mu^{-1}=-\mu^{2} \nabla \mu
$$

and the weak coupling condition (8), the electromagnetic force density (44) is recast in terms of the intensive electromagnetic fields $\mathbf{E}$ and $\mathbf{B}$ and the susceptibility tensors $\chi_{e}, \chi_{m}$ and $\chi_{\alpha}$ as

$$
\mathbf{f}^{\mathrm{em}}=-\mathbf{f}^{\mathrm{e}}-\mathbf{f}^{\mathrm{m}}-\mathbf{f}^{\alpha},
$$

with

$$
\begin{aligned}
\mathbf{f}^{\mathrm{e}} & =\frac{1}{2} \varepsilon_{0}(\mathbf{E} \odot \mathbf{E}) \boldsymbol{\nabla} \boldsymbol{\chi}_{e}, \\
\mathbf{f}^{\mathrm{m}} & =\frac{1}{2} \varepsilon_{0}(c \mathbf{B} \odot c \mathbf{B}) \boldsymbol{\nabla} \boldsymbol{\chi}_{m}, \\
\mathbf{f}^{\alpha} & =\varepsilon_{0}(\mathbf{E} \otimes c \mathbf{B}) \boldsymbol{\nabla} \boldsymbol{\chi}_{\alpha},
\end{aligned}
$$

where $\mathbf{f}^{\mathrm{e}}$ and $\mathbf{f}^{\mathrm{m}}$ are respectively the electric and magnetic ponderomotive force densities, and $\mathbf{f}^{\alpha}$ is the magnetoelectric ponderomotive force density. The negative signs in the relation (46) is due to the fact that the electromagnetic force density $\mathbf{f}^{\mathrm{em}}$ is defined as a force density exerted by the electromagnetic fields on the matter fields whereas the ponderomotive force densities $\mathbf{f}^{\mathrm{e}}, \mathbf{f}^{\mathrm{m}}$ and $\mathbf{f}^{\alpha}$ are defined as force densities resulting from the response of inhomogeneous matter fields to the presence of electromagnetic fields. In particular, the magnetoelectric ponderomotive force density $\mathbf{f}^{\alpha}$ results from the response of the inhomogeneous magnetoelectric susceptibility tensor $\chi_{\alpha}(n, \mathbf{u})$ to the presence of the electromagnetic fields $\mathbf{E}$ and B.

Since, the magnetoelectric susceptibility tensor $\chi_{\alpha}(n, \mathrm{u})$ is a function of the matter fields $n$ and $\mathrm{u}$, we predict that spatial inhomogeneities in a matter continuum that exhibits a linear magnetoelectric coupling in the presence of uniform electromagnetic fields $\mathbf{E}$ and $\mathbf{B}$ generates a magnetoelectric ponderomotive force. We believe that the existence of such a force has never been established before. In order to verify the existence of the magnetoelectric ponderomotive force, we suggest 
the following experiment. An inhomogeneous magnetoelectric sample is placed on a cantilever in an electric field $\mathbf{E}$ oscillating at an angular frequency $\omega_{1}$ and in a magnetic induction field $\mathbf{B}$ oscillating at an angular frequency $\omega_{2}$, i.e.

$$
\begin{aligned}
& \mathbf{E}=\mathbf{E}_{0} e^{i \omega_{1} t}, \\
& \mathbf{B}=\mathbf{B}_{0} e^{i \omega_{2} t} .
\end{aligned}
$$

For such a set-up, the definition (46) of the magnetoelectric ponderomotive force density predicts that the cantilever should detect a force oscillating at an angular frequency $\omega=\omega_{1}+\omega_{2}$.

\section{Conclusion}

By splitting the continuity equation (33) for the electromagnetic momentum (28), in the nonrelativistic limit, into a matter part (31) and an electromagnetic part (32), and using Maxwell's equations (12), (13), (18) and (19), and the Legendre transform (38) of the internal electromagnetic energy density, we determined the expression (46) for the electromagnetic force density. Thus, we obtained an explicit expression for the "magnetoelectric" ponderomotive force density $\mathbf{f}^{\alpha}$.

We also suggested an experimental verification of such a force by applying alternating electric and magnetic fields at distinct frequencies on an inhomogeneous material exhibiting a magnetoelectric coupling.

\section{Acknowledgments}

The authors would like to thank François A. Reuse and Charles E. Pfister for insightful discussions.

\section{References}

1. T. O'Dell, The Electrodynamics of Magneto-Electric Media (North Holland, Amsterdam, 1970).

2. A. Serdyukov, I. Semchenko, S. Tretyakov and A. Sihvola, Electromagnetics of BiAnisotropic Materials: Theory and Applications (Gordon and Breach, Amsterdam, 2001).

3. X.-L. Qi, T. L. Hughes and S.-C. Zhang, Phys. Rev. B 78 (2008) 195424.

4. D. Khomskii, Physics 2 (2009) 20.

5. M. Fiebig, Physics 4 (2011) 95.

6. H. Minkowski, Mathematische Annalen 68 (1910) 472.

7. L. D. Landau and E. M. Lifshits, The Electrodynamics of Continuous Media, 2nd edn. (Pergamon Press, Oxford, 1984).

8. E. C. G. Stückelberg von Breidenbach and P. B. Scheurer, Thermociétique Phénoménologique Galiléenne (Birkhauser, Basel, 1974).

9. W. Muschik, J. Theor. Appl. Mech. 50 (2012) 807.

10. F. A. Reuse, Electrodynamique (PPUR, Lausanne, 2012).

11. M. Abraham, Rendiconti del Circolo Matematico di Palermo 28 (1909) 1. 